FEDSM2013-16382

\title{
VORTEX PENALIZATION METHOD FOR SOLID-POROUS-FLUID MEDIA WITH APPLICATION TO PASSIVE FLOW CONTROL
}

\author{
Chloé Mimeau \\ Univ. Grenoble-Alpes, LJK \\ 51 , rue des Mathématiques \\ F-38041 Grenoble FRANCE \\ Chloe.Mimeau@imag.fr
}

\author{
Iraj Mortazavi \\ IMB Université de Bordeaux \\ $M C^{2}$ INRIA Bordeaux Sud-Ouest \\ 351, cours de la Libération \\ F-33405 Talence FRANCE \\ Iraj.Mortazavi@math.u-bordeaux1.fr
}

\author{
Georges-Henri Cottet \\ Univ. Grenoble-Alpes, LJK \\ 51 , rue des Mathématiques \\ F-38041 Grenoble FRANCE \\ Georges-Henri.Cottet@imag.fr
}

\begin{abstract}
In this work, a coupling of vortex methods with penalization methods is proposed in order to accurately and easily handle solid-fluid-porous media. This immersed boundary approach indeed maintains the efficiency and the robustness of vortex methods and allows to model the three different media without prescribing any boundary condition. In this paper, we propose an application of this immersed boundary method to passive flow control around a semi-circular cylinder, realized adding a porous sheath on the obstacle surface in order to smooth the flow dynamics.
\end{abstract}

\section{NOMENCLATURE}

$C_{D}$ drag coefficient

$D$ computational domain

$F, S$ fluid domain and solid domain

$F_{D}$ drag force

$\mathrm{Re}$ Reynolds number

$Z$ enstrophy

$d$ non-dimensional diameter

$h$ reference mesh size

$k$ intrinsic permeability

$l_{\text {ref }}$ height of the obstacle

$u_{\text {ref }}$ reference velocity

$\mathbf{u}=(u, v) \quad$ velocity field

$\mathbf{u}_{\mathbf{s}}$ body rigid motion $\overline{\mathbf{u}}$ mean velocity magnitude

$\mathbf{u}_{\infty}$ free stream velocity

$\Gamma_{D}$ computational domain boundaries

$\Delta t$ time step

$\Phi$ porosity

$\lambda$ penalization parameter

$\mu$ dynamic viscosity

$v$ kinematic viscosity

$\rho$ density of the fluid

$\tau$ porous layer thickness

$\chi_{S}$ characteristic function

$\omega$ vorticity field

\section{INTRODUCTION}

Vortex methods and penalization methods have been separately used to simulate high Reynolds number recirculating flows around obstacles. In this work, a hybrid particle-penalization technique is proposed in order to cover the advantages of both approaches. Here, the vortex method is used to approximate the penalized Vorticity Transport Equations (VTE). On one hand this technique maintains the efficiency and the robustness of vortex methods for turbulent Reynolds numbers focusing the computational task on the rotational zones and solving the flow equations in a fast Lagrangian way. On the other hand the penalization technique overcomes the difficulty of the vortex methods to satisfy accurately the no-slip boundary conditions. Here, the idea is 
to extend the fluid velocity inside the solid body and to solve the flow equations with a penalization term, depending on the intrinsic permeability, to enforce rigid motion inside the solid using a vorticity formulation. Moreover, this method eases numerical implementation since it enables to consider the governing equations in the whole computational domain allowing the use of structural grids and avoiding the overhead and the complexity typical of body fitted grids. Finally, and this is the main issue of this paper, the penalization approach allows to handle solidfluid-porous media where the differentiation between the three different media is easily performed defining the value of the penalization parameter without prescribing a boundary condition at the solid boundary or a condition at the porous-fluid interface. Here, the solid-porous-fluid configuration is applied to cover a semi-circular cylinder geometry with a porous coating in order to perform passive flow control. After performing convergence studies, a parametric study is carried out to determine the most efficient permeabilities in terms of flow control at transitional $(\operatorname{Re}=550)$ and highly transitional regime $(\operatorname{Re}=3000)$.

\section{VORTICITY FORMULATION AND VORTEX METHODS}

In this work, flow simulations are based on particle methods. The fluid particles which are displaced by convection and diffusion are characterized by their position and their vorticity. The vorticity transport is expressed by the Helmholtz equation (or Vorticity Transport Equation), obtained taking the curl of the incompressible Navier-Stokes equations and given in 2D by

$$
\frac{\partial \omega}{\partial t}+\mathbf{u} \cdot \nabla \omega=\frac{1}{\operatorname{Re}} \Delta \omega \text { in } D,
$$

where $\omega, \mathbf{u}$ and Re respectively denote the vorticity, the velocity and the Reynolds number. The Poisson equation

$$
\nabla^{2} \mathbf{u}=-\nabla \times \omega
$$

obtained from continuity equation, enables to recover velocity field once the vorticity field is known. The previous equations are approximated using a Vortex method [1,2]. These methods are very robust and low-cost to simulate high Reynolds number recirculating flows (see for example $[3,4]$ ). In this kind of approach, the VTE equation (Eq. 1) is solved using a two-fractional step (or viscous splitting) method. It relies on approximating separately the diffusion and convection terms at each time step. The convective part is solved using a "Vortex-In-Cell (VIC)" method (see e.g. [2]) with a semi-Lagrangian resolution. In this fractional step a convective velocity is associated to each finite vortex element through a high order interpolation procedure, and the displacement is achieved using a Runge-Kutta method. In order to avoid Lagrangian distortion, particles are then remeshed on the original grid using the same interpolation kernel as the one used previously to interpolate grid velocity values onto the particles. Finally, for computational efficiency and accuracy, diffusion and Poisson equation are solved on the grid using Fast Fourier Transforms (FFT).

\section{VORTEX PENALIZATION METHOD IN VORTICITY FOR- MULATION}

Before all, we show how the penalization method can be used successfully to model the flow of an incompressible fluid around an obstacle [5]. In the penalization technique the system is considered as a single flow, subject to the Navier-Stokes equation with a penalization term that enforces continuity at the solidfluid interface and rigid motion inside the solid. In this work, the penalization term is expressed using vorticity formulation. The main interest of the penalized vorticity formulation is that it replaces the usual vorticity creation algorithm in order to satisfy the no-slip boundary condition for vortex methods. This new technique avoids the convergence difficulties due to the particle creation on the solid boundaries (see [6] and [7]). We solve simultaneously the Brinkman equations in the solid and the NavierStokes equations in the fluid, considering whole the domain as a porous medium with zero (solid) or infinite permeabilities (fluid). Thus, defining the Reynolds number as $\operatorname{Re}=u_{\text {ref }} l_{r e f} / v$, the nondimensional penalized vorticity equation (or Brinkman-NavierStokes equation) reads

$$
\frac{\partial \omega}{\partial t}+(\mathbf{u} . \nabla) \omega=\frac{1}{\operatorname{Re}} \Delta \omega+\nabla \times\left[\lambda \chi_{S}\left(\mathbf{u}_{\mathbf{s}}-\mathbf{u}\right)\right]
$$

where $\chi_{S}$ denotes the characteristic function that yields 0 in the fluid and 1 in the solid, $\mathbf{u}_{\mathbf{s}}$ indicates the rigid body velocity which is zero in all this work since the body is fixed and $\lambda=\mu \Phi l_{\text {ref }} / \rho k u_{\text {ref }}$ is the non-dimensional penalization parameter, in inverse proportion to the permeability of the medium (with $k$ the intrinsic permeability, $\mu$ the viscosity, $\Phi$ the porosity of the porous material, $l_{\text {ref }}$ the height of the obstacle, $\rho$ the density and $u_{r e f}$ the reference velocity). The main advantage of this method is that it needs neither the mesh to fit the boundaries nor to specify no-slip boundary conditions. In addition it allows to compute the pressure as a continuous field on the whole domain including the solids.

The zone variation is realized changing the penalization coefficient that defines the permeability of each region. $\mathrm{Nu}$ merically, the fluid is considered as a porous medium with a very high permeability $(\lambda=0)$ and the bodies are considered as porous media with a very small permeability $\left(\lambda=10^{8}\right)$.

To discretize the penalized vorticity equation (Eq. 3) in a vortex method, the equation is split in substeps. At each time 
step, one successively solves the following equations

$$
\begin{array}{r}
\frac{\partial \omega}{\partial t}=\nabla \times\left(\lambda \chi_{S}\left(\mathbf{u}_{\mathbf{s}}-\mathbf{u}\right)\right) \\
\frac{\partial \omega}{\partial t}+(\mathbf{u} \cdot \nabla) \omega=0 \\
\frac{\partial \omega}{\partial t}=\frac{1}{R e} \Delta \omega
\end{array}
$$

To solve Eq. 4 we use an implicit scheme ( [6]) and we set

$$
\widetilde{\omega}^{n+1}=\nabla \times\left[\frac{\mathbf{u}^{n}+\lambda \Delta t \chi_{S} \mathbf{u}_{\mathbf{s}}{ }^{n}}{1+\lambda \Delta t \chi_{S}}\right] .
$$

where $\Delta t$ is the time step. The right hand side above is evaluated by second order centered finite differences.

At this stage, grid vorticity above a certain cut-off is used to create particle at grid point locations and Eq. 5 is solved by a classical vortex-in-cell method [2]. Particles are pushed with a RK4 time-stepping and are then remeshed on the original grid using the third order interpolation kernel $M_{4}^{\prime}$ [8]. Then, diffusion (Eq. 6) and Poisson equation (Eq. 2) are solved on the grid using FFT-based evaluations as described in [9]. Grid values for vorticity and velocity are now available for time $t_{n+1}$ and a new cycle of iterations can start. Moreover, the no-slip boundary conditions are naturally satisfied penalizing the vorticity transport equations.

This hybrid vortex penalization method has already been successfully used to simulate transitional and turbulent flows past bluff bodies [10,11].

\section{VORTEX PENALIZATION METHOD FOR SOLID- POROUS-FLUID MEDIA}

This section is devoted to the study of flows in solid-porousfluid media and aims to highlight the efficiency of the vortex penalization method to solve such problems. For three different solid, porous and fluid media configurations, it is of great importance to clearly understand the flow behavior at the fluid-porous interface in order to model the physics correctly. As described in [12], we can consider five different flow regions from the solid to the free flow in the fluid. The first one is the boundary layer inside the porous medium, close to the solid wall. This one is very thin compared to the second region which is characterized by the homogeneous porous flow with Darcy velocity (numbers 1 and 2 in Fig. 1). In the vicinity of the porous-fluid interface, two transient layers can be recognized (numbers 3 and 4 in Fig. 1). The first one corresponds to the increase of the porous layer velocity reaching $\mathbf{u}_{i}$ value at the interface and the second one to the fluid boundary layer standing from the interface to the free flow. The

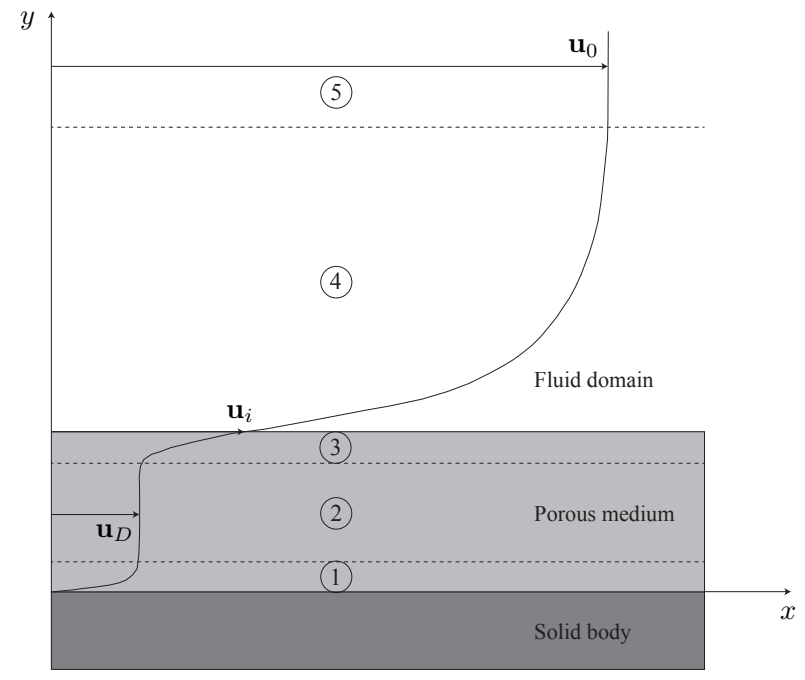

FIGURE 1: VELOCITY PROFILE IN THE VICINITY OF A POROUS MEDIUM.

fluid boundary layer growth is then determined by $\mathbf{u}_{0}-\mathbf{u}_{i}$ where $\mathbf{u}_{0}$ denotes the velocity of the main fluid flow (number 5 in Fig. 1). The aim is thus to find out a way to solve the flow both in the porous medium, the fluid region and at the interface between the two media. Several approaches have already been proposed to handle this problem like avoiding to solve the porous flow enforcing appropriate porous-fluid boundary conditions [13] or solving the governing equations of each region coupling Darcy equations and Navier-Stokes equations with a right treatment at the interface $[14,15]$. Nevertheless, these two approaches are not satisfactory since the first one neglects the porous medium physics and does not permit to have overall view of the problem and the second one is particularly difficult to handle especially because of the interface problem. The method presented here, based on the vortex-penalization technique, thus appears as a very good alternative since it involves a unique equation (Eq. 3) for the whole domain and ensures an accurate modeling of each of the different regions thanks to the dimensionless penalization factor $\lambda$. As mentioned in the previous section, $\lambda$ is expressed as $\lambda=\mu \Phi l_{\text {ref }} / \rho k u_{\text {ref }}$ where $l_{\text {ref }}, \rho, u_{r e f}=1$ in this study and the porosity $\Phi$ is close to 1 as imposed by Brinkman equations [16]. Therefore $\lambda$ essentially depends, in the inverse proportion, on the intrinsic permeability $k$ of the medium. Varying the $\lambda$ value thus directly defines the different media according to the following equation obtained using implicit Euler scheme for the penalization velocity discretization

$$
\widetilde{\mathbf{u}}^{n+1}=\frac{\mathbf{u}^{n}}{1+\lambda \Delta t \chi_{S}} .
$$


Indeed, in the fluid, the intrinsic permeability coefficient $k$ goes to infinity, thus the fluid can be considered numerically as a porous media with a very high permeability. We set $\lambda=0$ in this region. As a consequence, according to Eq. 8, the velocity in the fluid is not penalized $\left(\widetilde{\mathbf{u}}^{n+1}=\mathbf{u}^{n}\right)$ and since $\lambda=0$, the penalization term vanishes in Eq. 3, and we naturally recover the Vorticity Transport Equation (Eq. 1). On the contrary, the solid has a permeability coefficient $k$ which goes to zero, it can be consequently modeled setting the penalization parameter $\lambda$ to a very high value. In this study $\lambda$ equals $10^{8}$ in the solid, which vanishes the flow velocity in this region according to Eq. $8\left(\widetilde{\mathbf{u}}^{n+1} \rightarrow 0\right)$.

It was proved in [5] that solving Eq. 3 with such a value of $\lambda$ was equivalent to solve Darcy's law in the solid. Furthermore, setting the $\lambda$ parameter to an intermediate value, reasonably chosen between these two extreme values $\left(\lambda=0\right.$ and $\left.\lambda=10^{8}\right)$, would model a porous medium in which the flow has a Darcy velocity $\mathbf{u}_{D}$ (Fig. 1). As a conclusion, the variation of $\lambda$ corresponds to the variation of $k$ that specifies the intrinsic porous material permeability. The accuracy and efficiency of the penalization method come from its capability to take into account these variations of $\lambda$ and to capture the induced steep velocity variations at the different interfaces with a minimum number of discretization points.

\section{APPLICATION TO PASSIVE FLOW CONTROL}

Modeling the physics of three different regions enables one to deal with engineering problems involving porous media. In the following, this approach is validated for a simple but significant passive flow control problem.

Here, the solid-porous-fluid configuration is applied to cover a semi-circular cylinder geometry with a porous coating. The latter is settled on the obstacle external surface in order to modify the vorticity generation of the boundary layer and the vortex shedding. In fact, the presence of a porous medium at the solid-fluid interface imposes a kind of mixed boundary condition intermediate between the no-slip and the slip one on the solid boundary [17]. As a result, the shear forces are decreased and the flow dynamics is smoothed [12,18-20]. This technique, which allows to keep the obstacle geometry unchanged, should reduce drag forces and vortex induced vibrations improving the aerodynamic properties of the obstacle. The semi-circular cylinder can be considered as a simplified section of an outside rear-view mirror of a car or a motor cycle. The mirrors, due to their spanwise position, indeed generate a non-negligible wake which interferes with the flow past vehicle sides. This accounts for a good motivation to perform flow control past such obstacles. As it was shown in $[21,22]$, a flow past a square back obstacle is not dominated by longitudinal and hairpin three-dimensional vortical structures, therefore a preliminary two-dimensional study can be useful to supply information on general trends for a control.

The subsequent flow control simulations are performed at

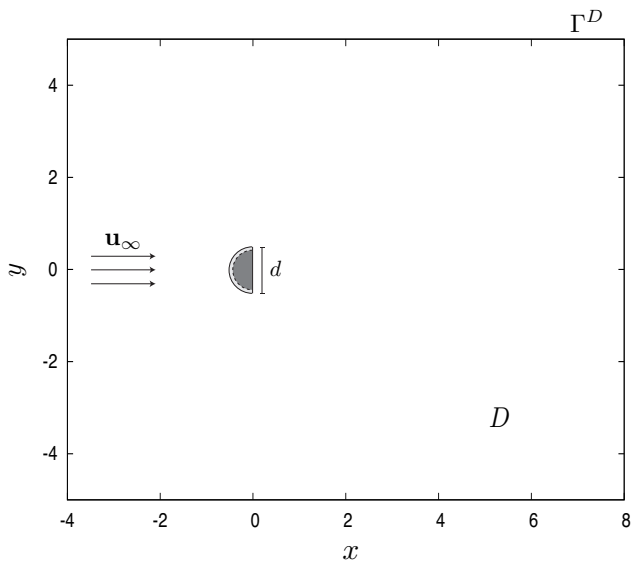

FIGURE 2: COMPUTATIONAL DOMAIN.

transitional $(\mathrm{Re}=550)$ and highly transitional regime $(\mathrm{Re}=$ 3000). As at these two regimes the flow is not turbulent, Direct Numerical Simulations (DNS) are performed to numerically solve Eq. 3. The semi-circular cylinder has a total dimensionless diameter of $d=1$ including a porous layer of thickness $\tau$ and whose back wall is centered at $(x, y)=(0,0)$ in the computational domain $D=[-4,8] \times[-5,5]$ (Fig. 2). The whole computational domain is meshed by an equispaced Cartesian orthogonal grid. As we use FFT-based evaluations to solve diffusion and Poisson equation, periodic boundary conditions are considered on the box walls $\Gamma_{D}$ and a correction of velocity is performed at each time step in order to satisfy the free stream velocity $\mathbf{u}_{\infty}=\left(u_{r e f}, 0\right)=(1,0)$ imposed at the inlet.

\section{Grid convergence}

First of all we perform a grid convergence study of the vortex-penalization method for flow past a solid semi-circular cylinder at $\mathrm{Re}=550$ and $\mathrm{Re}=3000$. This grid convergence is performed on three grid levels from res $=1200 \times 1000$ to res $=4800 \times$ 4000 in computational domain $D$. The results are reported in Table 1, giving the mean values of drag and enstrophy $Z=$ $\int_{D}|\omega|^{2} \mathrm{~d} \mathbf{x}$. At $\mathrm{Re}=550$, as the results on the two finest consecutive grids are very close (Table 1), the resolution therefore adopted for the further flow control simulations is $2400 \times$ $2000(h=0.005)$. The finest grid resolution $4800 \times 4000(h=$ $0.0025)$ is retained for the $\mathrm{Re}=3000$ case.

\section{Variation of the penalization parameter $\lambda$ for medium definition}

In this section, a $\lambda$-convergence study is carried out in order to determine the values of the penalization parameter corresponding to permeabilities at $\operatorname{Re}=550$ and $\operatorname{Re}=3000$. This study is performed ranging the value of $\lambda$ inside the whole semi- 
TABLE 1: MEAN VALUES OF DRAG COEFFICIENT $\left(C_{D}\right)$ AND ENSTROPHY ( $Z$ ) FOR FLOW PAST A SEMICIRCULAR CYLINDER AT Re=550 AND Re=3000.

\begin{tabular}{lllll}
\hline & \multicolumn{2}{r}{$\mathrm{Re}=550$} & \multicolumn{2}{r}{$\mathrm{Re}=3000$} \\
\hline Grid & $C_{D}$ & $Z$ & $C_{D}$ & $Z$ \\
\hline $1200 \times 1000(h=0.01)$ & 1.49 & 122 & 1.39 & 216 \\
$2400 \times 2000(h=0.005)$ & 1.91 & 158 & 1.64 & 294 \\
$4800 \times 4000(h=0.0025)$ & 1.98 & 161 & 1.86 & 301 \\
\hline
\end{tabular}
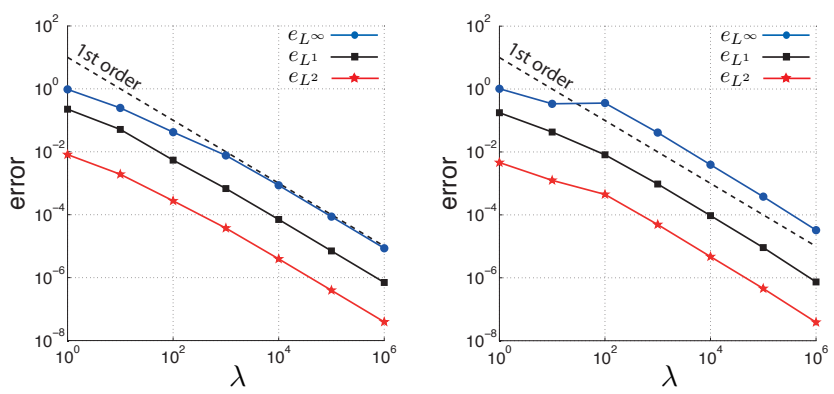

FIGURE 3: $\lambda$-CONVERGENCE STUDY FOR FLOW PAST A SEMI-CIRCULAR CYLINDER : THE ERRORS $e_{L^{\infty}}, e_{L^{1}}$ AND $e_{L^{2}}$ ARE PLOTTED AGAINST $\lambda$ VALUES AT Re $=550$ $(\mathrm{LEFT}) \mathrm{AND} \mathrm{Re}=3000(\mathrm{RIGHT})$

circular cylinder from 1 to $10^{6}$ with $10^{8}$ as reference case (solid case). In Fig. 3 the $L^{1}, L^{2}$ and $L^{\infty}$ norms of the error $e(y)$ of the mean velocity magnitude profile $|\overline{\mathbf{u}}|($ at $x=0)$ with respect to the solid case are plotted.

$$
\begin{aligned}
& e(y)_{L^{1}}=\left.\int_{y=-5}^{y=5}|| \overline{\mathbf{u}}\right|_{\lambda=10^{8}}(y)-|\overline{\mathbf{u}}|(y) \mid \mathrm{d} y \\
& e(y)_{L^{2}}=\left(\left.\int_{y=-5}^{y=5}|| \overline{\mathbf{u}}\right|_{\lambda=10^{8}}(y)-\left.|\overline{\mathbf{u}}|(y)\right|^{2} \mathrm{~d} y\right)^{1 / 2} \\
& e(y)_{L^{\infty}}=\left.\sup _{y \in[-5,5]}|| \overline{\mathbf{u}}\right|_{\lambda=10^{8}}(y)-|\overline{\mathbf{u}}|(y) \mid
\end{aligned}
$$

As Fig. 3 shows, the method has a first order convergence. This result is in agreement with our numerics since the penalization term (Eq. 4) is integrated using an implicit Euler scheme (Eq. 7 and 8). Moreover, we can see on this figure that for both regimes the semi-cylinder reaches the solid state from $\lambda=10^{5}$, where $e(y) \leq 10^{-4}$. Thus, a porous area can be defined with $\lambda$-values between 1 and $10^{4}$ with very low permeability for the latter one. We will respectively refer to the solid case and to the fluid case when $\lambda=10^{8}$ and $\lambda=0$ inside the layer.

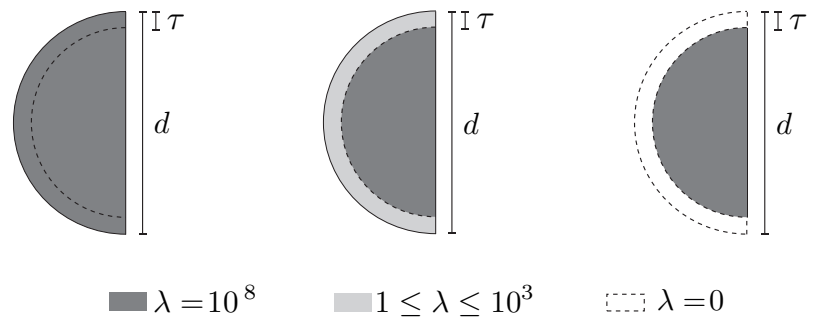

FIGURE 4: (LEFT) UNCONTROLLED/SOLID CASE, (CENTER) POROUS CASE, (RIGHT) FLUID CASE.

\section{Numerical results}

This section relies on the influence of the added porous layer permeability on the flow control behaviour and the efficiency of such a passive control. This parametric study is performed at $\mathrm{Re}=550$ and $\mathrm{Re}=3000$ considering four consecutive values of the porous permeability inside the layer, namely $\lambda=1$ (high permeability), $10,10^{2}, 10^{3}$ (low permeability) and comparing the results to the solid $\left(\lambda=10^{8}\right)$ and fluid $(\lambda=0)$ cases. The thickness of the coating is set to $\tau=10 \% d=0.1$ in each case (Fig. 4). According to the grid convergence (Table 1), the numerical simulations carried out at $\operatorname{Re}=550$ and $\operatorname{Re}=3000$ are respectively performed on grid $2400 \times 2000(h=0.005)$ and grid $4800 \times 4000(h=0.0025)$ in computational domain $D$.

In order to analyze the effects of our control approach we compare global flow quantities like the drag force $\left(F_{D}\right)$, computed according to the momentum equation [23] and the enstrophy $(Z)$, expressed as the integral of the square of the vorticity, allowing to measure the dissipation effects in the flow as well as the delay of transition to turbulence. Note that in this study we consider drag force $\left(F_{D}\right)$ instead of drag coefficient $\left(C_{D}\right)$ since the computation of the latter involves the diameter $d$ of the obstacle which is not clearly defined because of the porous coating.

As can be seen in Fig. 5, which represents dimensionless time history of global flow quantities at $\operatorname{Re}=550$, setting $\lambda=1$ inside the layer clearly appears as the best solution in terms of flow regularization. Indeed, the mean value of drag force (Fig. 5 (up)) reaches for $\lambda=1$ an optimum value close to the one of the fluid case, showing a drag reduction of about $30 \%$ compared to uncontrolled case. For all the other values of porous $\lambda$, the drag reduction effects are nearly nonexistent. Results of enstrophy evolution (Fig. 5 (bottom)) show a progressive reduction of the dissipation effects and the delay to transition with the decrease of the $\lambda$-value. Furthermore, we note that the result obtained with $\lambda=1$ is even better than the one of fluid case and represents an improvement of nearly $40 \%$ compared to uncontrolled case. These quantitative results are confirmed by the mean vorticity and velocity fields showing the smoothing of wake dynamics generated by the presence of the highly permeable layer. Indeed, with $\lambda=1$ the transversal dimension of the wake is smaller, the 

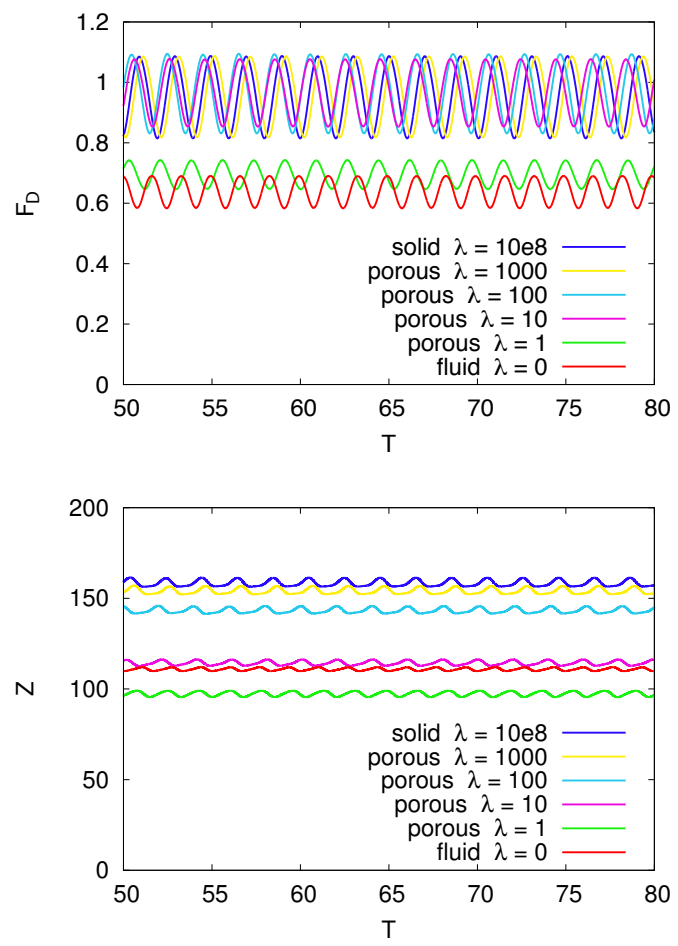

FIGURE 5: EFFECTS OF VARIOUS LAYER PERMEABILITIES ON DRAG FORCE (UP) AND ENSTROPHY (BOTTOM) RELATED TO FLOW PAST A SEMI-CIRCULAR CYLINDER $\mathrm{AT} \operatorname{Re}=550$.
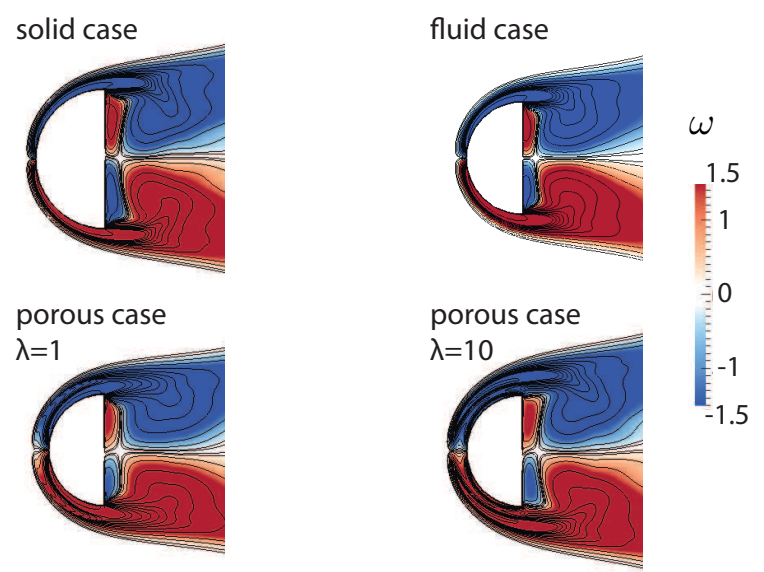

FIGURE 6: ZOOM OF THE MEAN VORTICITY FIELDS AND ISOLINES FOR THE FLOW PAST A SEMI-CIRCULAR CYLINDER AT Re $=550$.
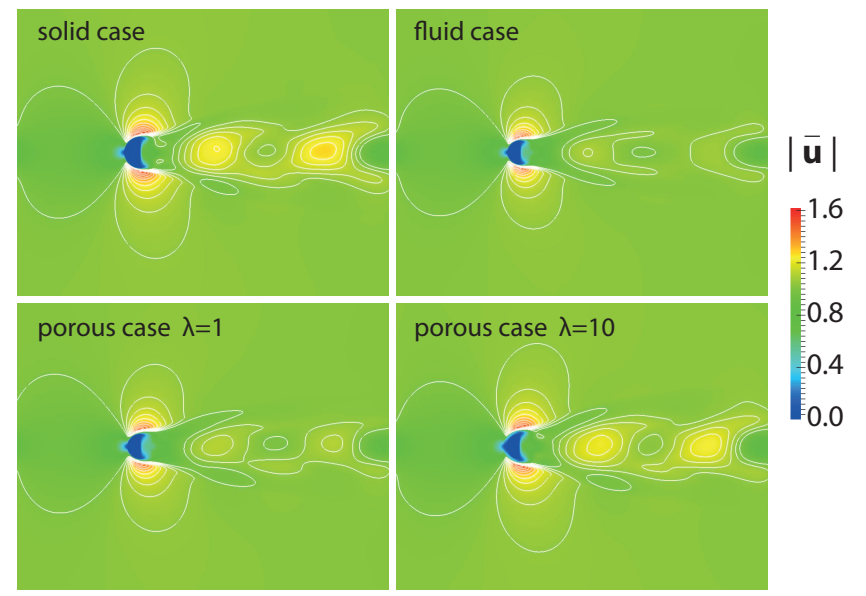

FIGURE 7: FIELDS AND ISOLINES OF MEAN VELOCITY MAGNITUDE FOR THE FLOW PAST A SEMI-CIRCULAR CYLINDER AT $\operatorname{Re}=550$.

back recirculation zone is sharply reduced (Fig. 6) and the vortices swirl with lower velocity (Fig. 7).

At $\mathrm{Re}=3000$ the best solution for global flow regularization is also achieved setting the $\lambda$ parameter to 1 inside the porous coating. This configuration leads to a drag and enstrophy reduction of respectively $21 \%$ and $44 \%$ compared to the uncontrolled case (Fig. 8) and shows a drastic decrease of the flow particles velocity (Fig. 10). In terms of control effects, the main difference one can notice here in comparison to the parameter study performed at transitional regime concerns the flow behaviour observed when $\lambda=10$ inside the layer. Contrary to the other $\lambda$ values, the flow obtained with the latter is non-periodic (Fig. 8). This flow irregularity impacts the mean drag value, which is increased of about $10 \%$ compared to the uncontrolled case. Figure 10 also confirms the negative effects of the non-periodicity of the flow showing high particles velocity with a chaotic distribution of the vortices as well as an extension of the transversal dimension of the wake (Fig. 9).

\section{CONCLUSION}

In this work, a hybrid vortex-penalization method was proposed in order to easily handle solid-fluid-porous media. The differentiation between the three different media is indeed performed defining the value of the penalization parameter in the Brinkman-Navier-Stokes equations without prescribing a boundary condition at the solid boundary or a condition at the porousfluid interface. An application to passive flow control past a semi-circular cylinder was performed at transitional and highly transitional regimes, consisting in adding a porous sheath on the obstacle surface in order to smooth the flow dynamics. The para- 

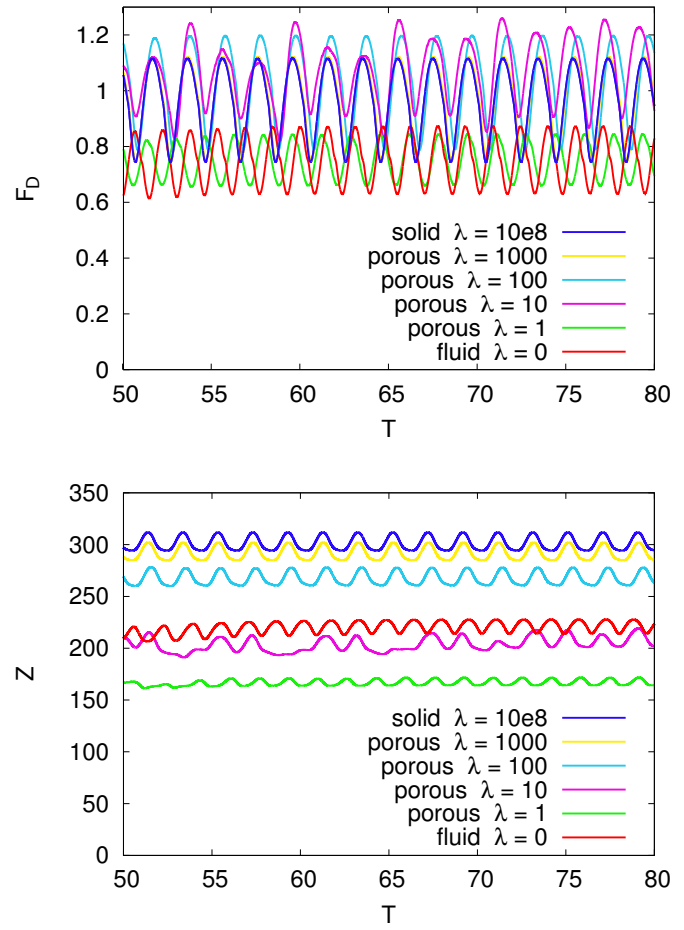

FIGURE 8: EFFECTS OF VARIOUS LAYER PERMEABILITIES ON DRAG FORCE (UP) AND ENSTROPHY (BOTTOM) RELATED TO FLOW PAST A SEMI-CIRCULAR CYLINDER $\mathrm{AT} \operatorname{Re}=3000$.
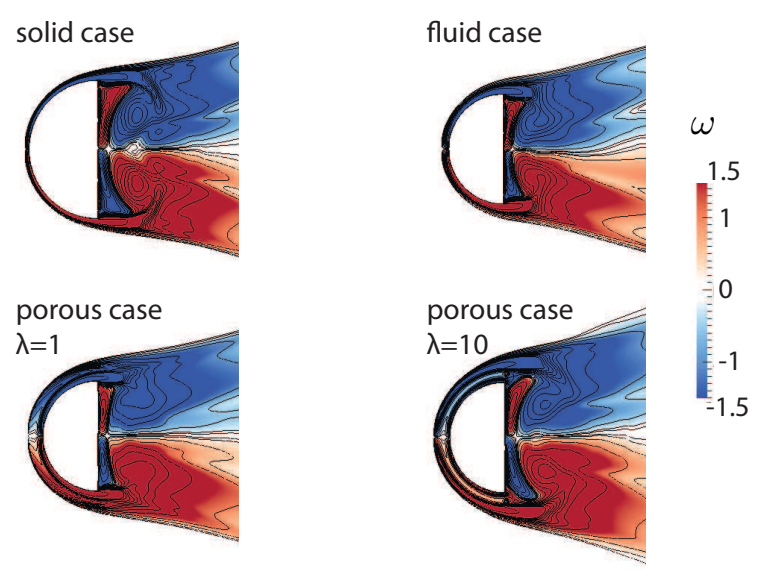

FIGURE 9: ZOOM OF THE MEAN VORTICITY FIELDS AND ISOLINES FOR THE FLOW PAST A SEMI-CIRCULAR CYLINDER AT Re $=3000$.

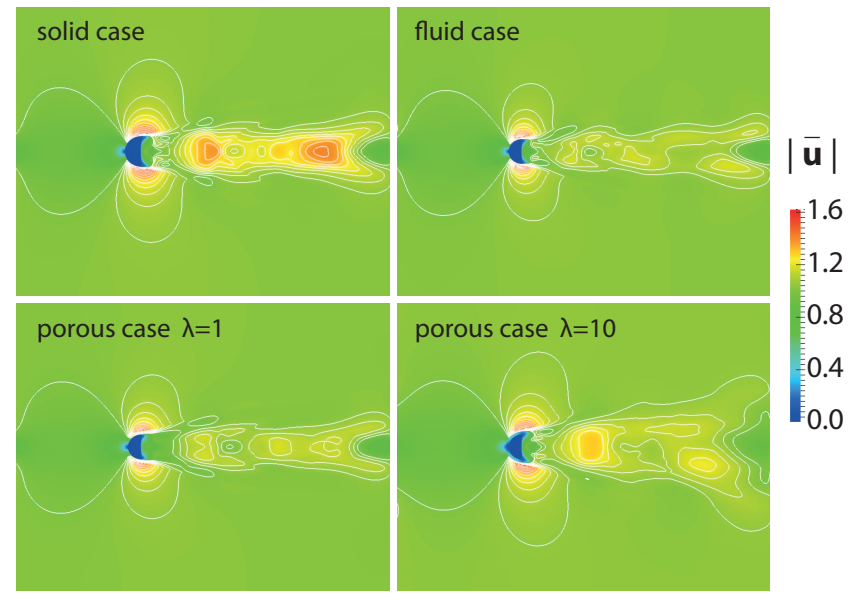

FIGURE 10: FIELDS AND ISOLINES OF MEAN VELOCITY MAGNITUDE FOR THE FLOW PAST A SEMI-CIRCULAR CYLINDER AT Re $=3000$.

metric study carried out in the last part of this paper, shown that the best results in terms of passive flow control were achieved using highly porous layers. The presence of a very permeable coating at the solid-fluid interface is indeed responsible for a decrease of the shear forces and the vorticity generation of the boundary layer and was shown to lead to drag reductions of about $30 \%$ at $\operatorname{Re}=550$ and $20 \%$ at $\operatorname{Re}=3000$. Further studies will entail a passive control for high Reynolds flows (closer to real problems) and $3 \mathrm{D}$ cases.

\section{REFERENCES}

[1] Chorin, A. J., 1974. "Numerical Study of Slighly Viscous Flow". Journal of Fluid Mechanics, 57, pp. 785-796.

[2] Cottet, G. H., and Koumoutsakos, P. D., 2000. Vortex Methods - Theory and Practice. Cambridge University Press.

[3] Ghoniem, A. F., and Gagnon, Y., 1987. "Vortex Simulation of Laminar Recirculating Flows". Journal of Coтриtational Physics, 68, pp. 346-377.

[4] Sethian, J. A., and Ghoniem, A. F., 1988. "Validation Study of Vortex Methods". Journal of Computational Physics, 74, pp. 283-317.

[5] Angot, P., Bruneau, C. H., and Fabrie, P., 1999. "A Penalization Method to Take Into Account Obstacles in Incompressible Viscous Flows". Numerische Mathematik, 81, pp. 497-520.

[6] Coquerelle, M., and Cottet, G. H., 2008. "A Vortex Level Set Method for the Two-Way Coupling of an Incompressible Fluid with Collinding Rigid Bodies". Journal of Computational Physics, 227, pp. 9121-9137.

[7] Cottet, G. H., and Maitre, E., 2004. "A Level-Set Formu- 
lation of Immersed Boundary Methods for Fluid-Structure Interaction Problems". Comptes Rendus de l'Acadmie des Sciences de Paris, 338, pp. 51-56.

[8] Monaghan, J. J., 1985. "Extrapolating b-Splines for Interpolation". Journal of Computational Physics, 60, pp. 253262.

[9] Chatelain, P., and Koumoutsakos, P., 2010. "A FourierBased Elliptic Solver For Vortical Flows With Periodic and Unbounded Directions". Journal of Computational Physics, 229, pp. 2425-31.

[10] Cottet, G. H., Gallizio, F., Magni, A., and Mortazavi, I., 2010. "A Vortex Immersed Boundary Method for Bluff Body Flows". In Proceedings of 3rd Joint USEuropean ASME Fluids Engineering Summer Metting, August, 2010, ASME, pp. 2409-2414. Paper number FEDSMICNMM2010-30787.

[11] Cottet, G. H., Gallizio, F., Magni, A., and Mortazavi, I., 2011. "A Vortex Penalization Method for Flows with Moving Immersed Obstacles”. In Proceedings of ASME-JSMEKSME Joint Fluids Engineering Conference, July, 2011, ASME, pp. 3703-3708. Paper number AJK2011-20015.

[12] Bruneau, C. H., and Mortazavi, I., 2008. "Numerical Modelling and Passive Flow Control Using Porous Media". Computers \& Fluids, 37, pp. 488-498.

[13] Beavers, G. D., and Joseph, D. D., 1967. "Boundary Conditons at a Naturally Permeable Wall". Journal of Fluid Mechanics, 30, pp. 197-207.

[14] Breugem, W. P., Boersma, B. J., and Uittenbogaard, R. E., 2005. "The Laminar Boundary Layer Over a Permeable Wall”. Transport in Porous Media, 59, pp. 267-300.

[15] Hanspal, N. S., Waghode, A. N., Nassehi, V., and Wakeman, R. J., 2006. "Numerical Analysis of Coupled Stokes/Darcy Flows in Industrial Filtrations". Transport in Porous Media, 64, pp. 73-101.

[16] Nield, D. A., and Bejan, A., 1999. Convection in Porous Media. Springer.

[17] Carbou, G., 2008. "Brinkmann Model and Double Penalization Method for the Flow Around a Porous Thin Layer". Journal of Mathematical Fluid Mechanics, 10, pp. 126158.

[18] Bruneau, C. H., and Mortazavi, I., 2004. "Passive Control of the Flow Around a Square Cylinder Using Porous Media”. International Journal for Numerical Methods in Fluids, 46, pp. 415-433.

[19] Bruneau, C. H., and Mortazavi, I., 2006. "Control of Vortex Shedding Around a Pipe Section Using a Porous Sheat". Journal of Offshore and Polar Engineering, 16.

[20] Bruneau, C. H., Gilliéron, P., and Mortazavi, I., 2008. "Passive Control Around a Two-Dimensional Square Back Ahmed Body Using Porous Media". Journal of Fluids Engineering, 130.

[21] Rouméas, M., Gilliéron, P., and Kourta, A., 2009. “Analysis and Control of the Near-Wake Flow Over a Square-Back Geometry". Computers \& Fluids, 38, pp. 60-70.

[22] Bruneau, C. H., Creusé, E., Depeyras, D., Gilliéron, P., and Mortazavi, I., 2010. "Coupling Active and Passive Techniques to Control the Flow Past the Square Back Ahmed Body". Computers \& Fluids, 38, pp. 1875-1892.

[23] Noca, F., Shiels, D., and Jeon, D., 1999. "A Comparison of Methods for Evaluating Time-Dependent Fluid Dynamic Forces on Bodies, Using Only Velocity Fields and Their Derivatives". Journal of Fluids and Structures, 13, pp. 551-578. 